\title{
Influence of induction therapy, immunosuppressive regimen and anti-viral prophylaxis on development of lymphomas after heart transplantation: data from the spanish post-heart transplant tumour registry
}

\author{
Maria G. Crespo-Leiro, MD ${ }^{\mathrm{a}}$, Luis Alonso-Pulpón, MD ${ }^{\mathrm{b}}$, José M. Arizón, MD ${ }^{\mathrm{c}}$, \\ Luis Almenar, $\mathrm{MD}^{\mathrm{d}}$, Juan F. Delgado, $\mathrm{MD}^{\mathrm{e}}$, Jesús Palomo, $\mathrm{MD}^{\mathrm{f}}$, Nicolás Manito, \\ $\mathrm{MD}^{\mathrm{g}}$, Gregorio Rábago, $\mathrm{MD}^{\mathrm{h}}$, Ernesto Lage, $\mathrm{MD}^{\mathrm{i}}$, Beatriz Diaz, $\mathrm{MD}^{\mathrm{j}}$, Eulalia \\ Roig, $\mathrm{MD}^{\mathrm{k}}$, Domingo Pascual, MD ${ }^{1}$, Teresa Blasco, $\mathrm{MD}^{\mathrm{m}}$, Luis de la Fuente, \\ $\mathrm{MD}^{\mathrm{n}}$, Marta Campreciós, $\mathrm{MD}^{\mathrm{o}}$, José A. Vázquez de Prada, $\mathrm{MD}^{\mathrm{p}}$, Javier Muñiz, \\ $\mathrm{MD}^{\mathrm{q}}$
}

${ }^{a}$ Complejo Hospitalario Universitario Juan Canalejo, La Coruña, Spain

${ }^{b}$ Clínica Puerta de Hierro, Madrid, Spain

${ }^{c}$ Hospital Universitario Reina Sofía, Córdoba, Spain

${ }^{d}$ Hospital Universitario La Fe, Valencia, Spain

${ }^{e}$ Hospital Universitario 12 de Octubre, Madrid, Spain

${ }^{f}$ Hospital General Universitario Gregorio Marañón, Madrid, Spain

${ }^{g}$ Hospital Universitario de Bellvitge, Barcelona, Spain

${ }^{h}$ Clínica Universitaria de Navarra, Pamplona, Spain

${ }^{i}$ Hospital Universitario Virgen del Rocío, Sevilla, Spain

${ }^{j}$ Hospital Universitario Central de Asturias, Oviedo, Spain

${ }^{k}$ Hospital Clinic I Provincial, Barcelona, Spain

${ }^{l}$ Hospital Universitario Virgen de la Arrixaca, Murcia, Spain

${ }^{m}$ Hospital Universitario Miguel Servet, Zaragoza, Spain

${ }^{n}$ Hospital Clínico Universitario, Valladolid, Spain

${ }^{o}$ Hospital de la Santa Creu I Sant Pau, Barcelona, Spain

${ }^{p}$ Hospital Universitario Marqués de Valdecilla, Santander, Spain

${ }^{q}$ Instituto Ciencias de la Salud, Universidad de La Coruña, Spain.

\section{Resume}

Background. Lymphoma after heart transplantation (HT) has been associated with induction therapy and herpesvirus infection. It is not known whether anti-viral agents administered immediately after HT can reduce the incidence of lymphoma.

Methods. This study was a retrospective review of 3,393 patients who underwent HT in Spain between 1984 and December 2003. Variables examined included development of lymphoma and, as possible risk factors, recipient gender and age, induction therapies (anti-thymocyte globulin, OKT3 and anti-interleukin-2 receptor antibodies) and anti-viral prophylaxis (acyclovir or ganciclovir). To study the effect of evolving treatment strategy, three HT eras were considered: 1984 to 1995; 1996 to 2000; and 2001 to 2003.

Results. Induction therapy was employed in $>60 \%$ of HTs, and anti-viral prophylaxis in $>50 \%$. There were 62 cases of lymphoma (3.1 per 1,000 person-years, 95\% confidence interval: 2.4 to 4.0 ). Univariate analyses showed no influence of gender, age at transplant, HT era, pre-HT smoking or the immunosuppressive maintenance drugs used in the first 3 months post-HT. The induction agent anti-thymocyte globulin (ATG) was associated with increased risk of lymphoma, and prophylaxis with acyclovir with decreased risk of lymphoma. Multivariate analyses (controlling for age group, gender, pre-HT smoking and immunosuppression in the first 3 months with mycophenolate mofetil and/or tacrolimus) showed that induction increased the risk of lymphoma if anti-viral prophylaxis was not used (regardless of induction agent and anti-viral agent), but did not increase the risk if anti-viral prophylaxis was used.

Conclusions. Induction therapies with ATG or OKT3 do or do not increase the risk of lymphoma depending on whether anti-viral prophylaxis with acyclovir or ganciclovir is or is not employed, respectively. 
Post-transplant lymphoproliferative disorders (PTLDs) have been associated with Epstein-Barr virus (EBV) infection ${ }^{1,2}$ and with induction therapy. ${ }^{3}$ In the case of heart transplant $(\mathrm{HT})$ patients, induction with OKT3 has been associated with PTLD in some studies, ${ }^{4}$ but not in others. ${ }^{5-7}$ Induction is currently used in $40 \%$ to $50 \%$ of $\mathrm{HTs}^{8},{ }^{8}$ mainly with anti-thymocyte globulin (ATG) or antibodies against interleukin-2 receptor (IL-2R) rather than OKT3.

Anti-viral prophylaxis with acyclovir or ganciclovir was originally introduced mainly to combat cytomegalovirus. It presumably may also combat EBV and other viruses that may prove to be associated with PTLD, but whether it actually does has not been established. Thus, it is not known whether the efficacy of any such action is influenced by the use of induction therapy or itself influences any risk associated with induction therapy.

The Spanish Post-Heart Transplant Tumour Registry (SPHTTR) is singular in that its 18 collaborating centers - all the Spanish hospitals performing HTs - continually provide direct updates with data on tumors in all patients who have undergone HT in Spain since the initiation of heart transplantation in this country in 1984. In this investigation we report the incidence of postHT lymphoma among adult HT patients in Spain as well as the influence of immunosuppressive regimen, induction treatment and anti-viral prophylaxis. A fuller analysis that considers all neoplasias (not only lymphoma) will be published in due course.

\section{METHODS}

\section{Patients}

A total of 4,089 patients aged $>15$ years who underwent heart transplantation in Spain between 1984 and December 31, 2003 were identified in the SPHTTR. From these, we excluded the 695 patients who died in the first 3 months after HT, and 1 patient for whom the date of diagnosis of a first post-HT tumor had not been recorded. The remaining 3,393 patients were followed up to December 2004. Some $84.7 \%$ of these patients were men, with a mean age at time of transplantation of $51.4 \pm 11.0$ years and a median follow-up time of 5.2 years (mean $5.8 \pm 4.1$ years, maximum 20.2 years).

\section{Variables}

The outcome data analyzed included occurrence of post-HT lymphoma and date of diagnosis. The treatment variables considered were: use of induction therapy, and the agents employed for this purpose (OKT3, ATG, daclizumab, basiliximab); the agents used for maintenance immunosuppression (cyclosporine [CsA], tacrolimus, azathioprine, mycophenolate mofetil [MMF], everolimus, sirolimus); and the use of anti-viral prophylaxis, and the agents employed for this purpose (acyclovir in the first 3 months after HT, ganciclovir or either). Risk factors considered were pre-HT smoking (information on post-HT smoking was incomplete and therefore not evaluated) and age at HT ( $<45,45$ to 54,55 to $64, \geq 65$ years). With a view toward evaluating the effect of changes in immunosuppressive practice or HT protocols, the era in which HT had been performed was also introduced as an independent variable; the three eras considered were 1984 to 1997 (the pre-MMF era), 1998 to 2000 (the pre-IL-2R blocker MMF era) and 2001 to 2003 (the IL-2R blocker era). Information on EBV status of donor and recipient was available for only 920 patients (27\%), and was therefore excluded from the analyses.

This research was approved by the institutional review board of each participating center. 


\section{Statistical Analysis}

For each patient, the number of years at risk was defined as the time between the date of HT and December 2004 or death, whichever occurred first. The incidence of lymphoma per 1,000 person-years was estimated for each gender and age group, and for follow-up to 3 and 6 months and $1,2,3,4,5$ and 10 years. Univariate risk analyses were performed to estimate the influence of age group, gender, pre-HT smoking, HT era, MMF in the first 3 months, tacrolimus, induction therapy, induction with OKT3, induction with ATG, induction with basiliximab, induction with daclizumab, prophylaxis with acyclovir, prophylaxis with ganciclovir, and prophylaxis with either acyclovir or ganciclovir. Multivariate Poisson models were fitted in which the association of lymphoma with induction in general and with induction by OKT3 and by ATG was evaluated, while adjusting for age group, gender, pre-HT smoking and immunosuppression in the first 3 months with MMF and/or tacrolimus, in each of four groups defined on the basis of anti-viral prophylaxis: patients given acyclovir; patients not given acyclovir; patients given acyclovir or ganciclovir; and patients given neither acyclovir nor ganciclovir. The criterion for statistical significance was $p<0.05$.

\section{RESULTS}

Of the 3,393 patients included in this study, 1,801 (53.1\%) underwent HT in the pre-MMF era (1984 to 1997), 844 (24.9\%) in the intermediate era (1998 to 2000) and 748 (22\%) in the anti-IL2R antibodies era (2001 to 2003). Some $48.9 \%$ of patients had been smokers before HT.

Data on the use of individual immunosuppressive agents are listed in Table 1. Steroids were used by all patients in the first 3 months post-HT, and by $62.2 \%$ after 2 years. The use of CsA and azathioprine declined gradually with increasing time post-HT, whereas tacrolimus, MMF and sirolimus exhibited the reverse trend. OKT3, ATG, basiliximab and daclizumab were used almost exclusively in the first 3 months post-HT, presumably for induction therapy. On this basis, induction therapy was used in $60.5 \%$ of the patients. The duration of induction therapy was $7.8 \pm$ 3.9 days with OKT3, and $4.9 \pm 3.3$ days with ATG.

Acyclovir was administered to 1,369 patients $(40.3 \%)$ during the first 3 months post-HT, ganciclovir to 1,017 (30\%) during the first month, and either acyclovir or ganciclovir (or both) to $1,815(53.5 \%)$. A total of 62 lymphomas developed in 62 patients. The overall incidence of lymphomas per 1,000 person-years was 3.1 (95\% confidence interval [CI]: 2.4 to 4 ); incidence per 1,000 person-years was 4.6 at 1 year after HT ( $95 \%$ CI: 2.8 to 7.6 ), 2.7 after 5 years (95\% CI: 2 to 3.8 ) and 3 after 10 years (95\% CI: 2.3 to 3.9) (Table 2). The overall incidence was 3.1 per 1,000 person-years for both men and women $(p=0.948)$, and did not vary significantly among the four age groups at HT.

Univariate analyses (Table 3) showed no differences in incidence related to gender, age at transplant, HT era, pre-HT smoking, or immunosuppressive maintenance drugs used in the first 3 months. ATG was associated with increased risk (relative risk [RR] $=2.4,95 \%$ CI: 1.3 to $4.5 ; p=$ $0.004)$, and anti-viral prophylaxis with acyclovir exhibited borderline association with decreased risk $(\mathrm{RR}=0.6,95 \%$ CI: 0.3 to $1.0 ; p=0.05)$.

Multivariate analyses were performed in which the association of lymphoma with OKT3, ATG or induction in general was estimated in each of the four anti-viral prophylaxis groups distinguished (i.e., patients given acyclovir, patients not given acyclovir, patients given acyclovir or ganciclovir, patients given neither acyclovir nor ganciclovir) while controlling for age group, gender, pre-HT smoking and immunosuppression in the first 3 months with MMF and/or tacrolimus. These analyses showed that increased risk of lymphoma was associated with both ATG and OKT3 among patients who were not given acyclovir (RR $=3.6$ [95\% CI 1.6 to 7.9] for ATG, 2.2 [95\% CI: 1.4 to 7.0] for OKT3, 2.8 [95\% CI: 1.5 to 5.4] for induction in general) and among patients given neither acyclovir nor ganciclovir $(\mathrm{RR}=3.7$ [95\% CI: 1.6 to 8.3] for ATG, 2.7 [95\% CI: 1 to 7.4 ] for OKT3, 3.2 [95\% CI: 1.6 to 6.6] for induction in general), but was not 
associated with either agent among patients given acyclovir or patients given either acyclovir or ganciclovir (Figure 1).

Table 1. Demographics, Immunosuppressive Agents and Anti-viral Prophylaxis

\begin{tabular}{lc}
\hline Clinical characteristics & \\
\hline$n$ & \\
Males (\%) & 3,393 \\
Age (mean \pm SD) & 84.7 \\
Heart transplantation period (\%) & $51.4 \pm 11$ \\
1984-1997 & 53.1 \\
1998-2000 & 24.9 \\
2000-2003 & 22 \\
Basal immunosuppression (\%) & \\
Cyclosporine & 79.1 \\
Azathioprine & 60.5 \\
Tacrolimus & 8.3 \\
MMF & 25.6 \\
Sirolimus & 0.4 \\
Everolimus & 0.2 \\
OKT3 & 37.4 \\
ATG & 14.3 \\
Basiliximab & 5.6 \\
Daclizumab & 3.4 \\
Anti-viral prophylaxis (\%) & \\
Acyclovir (first 3 months) & 40.3 \\
Ganciclovir ( $\geq 1$ month) & 30 \\
Acyclovir or ganciclovir & 53.5 \\
& \\
\hline
\end{tabular}

ATG, anti-thymocyte globulin (also including thymoglobulin).

Table 2. Incidence of Lymphomas

\begin{tabular}{lccc}
\hline & \multicolumn{3}{c}{ Lymphomas } \\
\cline { 2 - 4 } & Person-years & Tumors & $\mathrm{IR}^{\mathrm{a}}(95 \% \mathrm{CI})^{\mathrm{b}}$ \\
\hline Overall & $19,885.1$ & 62 & $3.1(2.4-4)$ \\
By follow-up & & & \\
3 months & 847.3 & 3 & $3.5(1.1-11)$ \\
6 months & $1,676.1$ & 7 & $4.2(2-8.8)$ \\
1 year & $3,262.6$ & 15 & $4.6(2.8-7.6)$ \\
2 years & $6,161.7$ & 20 & $3.3(2.1-5)$ \\
3 years & $8,679.8$ & 22 & $2.5(1.7-3.9)$ \\
4 years & $10,888.3$ & 28 & $2.6(1.8-3.7)$ \\
5 years & $12,789.0$ & 35 & $2.7(2-3.8)$ \\
10 years & $18,308.1$ & 55 & $3(2.3-3.9)$ \\
& & & \\
\hline
\end{tabular}

${ }^{a}$ Incidence rate (IR) per 1,000 person-years.

${ }^{\mathrm{b}} \mathrm{CI}$, confidence interval. 
Table 3. Relative Risk (RR) of Lymphoma Associated With Various Factors (Univariate Analyses)

\begin{tabular}{lcc}
\hline & \multicolumn{2}{c}{ Lymphomas } \\
\cline { 2 - 3 } & RR $(95 \%$ CI $)$ & $p$-value \\
\hline Gender & & \\
Male & $1(0.5-2.1)$ & 0.9483 \\
$\quad$ Female & 1.00 & - \\
Age group & & - \\
$\quad$ <5 & 1.00 & 0.2577 \\
45-54 & $0.7(0.4-1.3)$ & 0.3879 \\
55-64 & $0.8(0.4-1.4)$ & 0.8414 \\
Z65 & $1.1(0.4-3.3)$ & 0.9324 \\
Smoker pre-HT & $1(0.6-1.7)$ & \\
HT period & & 0.8667 \\
1984-1997 & 1.00 & 0.5042 \\
1998-2000 & $0.9(0.5-1.8)$ & 0.6535 \\
2001-2003 & $0.7(0.3-2)$ & 0.7298 \\
MMF (yes/no) & $0.8(0.4-1.8)$ & 0.1497 \\
Tacrolimus (yes/no) & $0.8(0.2-3.2)$ & 0.5600 \\
Induction therapy & $1.5(0.9-2.5)$ & 0.0044 \\
OKT3 & $1.2(0.7-2.2)$ & 0.3163 \\
ATG 2.4 & $(1.3-4.5)$ & 0.4654 \\
Basiliximab & 0.00 & 0.0500 \\
Daclizumab & 0.00 & 0.1921 \\
Profilax & $0.6(0.3-1)$ & \\
Aciclovir & $0.9(0.5-1.6)$ & \\
Ganciclovir & $0.7(0.4-1.2)$ & \\
Aciclovir/ganciclovir & & \\
& & \\
\hline
\end{tabular}

CI, confidence interval; HT, heart transplantation; MMF, mycophenolate mofetil; ATG, anti-thymocyte globulin (also including thymoglobulin).

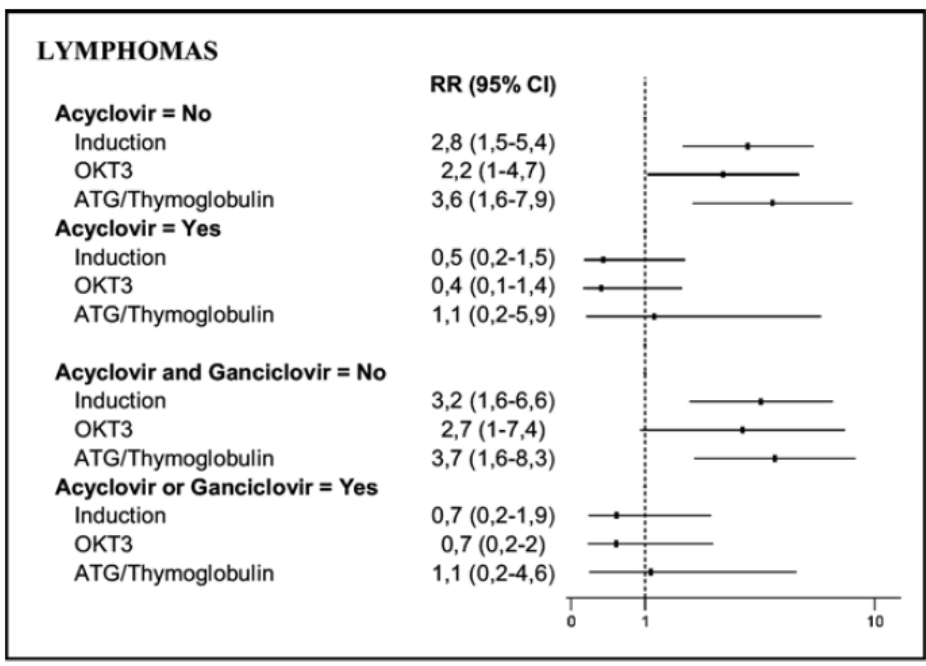

Figure 1. Effect of induction therapies on the risk of post-HT lymphomas for patients who were and were not given anti-viral prophylaxis with acyclovir, and for patients who were and were not given anti-viral prophylaxis with acyclovir or gancyclovir, after adjustment for gender, age group, pre-HT smoking and MMF or tacrolimus in the first 3 months. RR, relative risk; CI, confidence interval. 


\section{DISCUSSION}

This study reviewed 3,393 HT patients, $61 \%$ of whom had received induction therapy and 54\% anti-viral prophylaxis. Sixty-two patients developed lymphoma, representing an overall incidence of 3.1 per 1,000 person-years (95\% CI: 2.4 to 4). Overall incidence was not affected by gender or age at HT.

Among Spanish HT patients, the incidence of lymphoma per 1,000 person-years during successive 1-year periods has been greatest during the first year post-HT (as in Opelz et al's analysis of the CTS database ${ }^{9}$ ), but then fell and eventually leveled off at about 3.8, as can be calculated from the data in Table 2 .

The incidence of lymphoma was not affected by immunosuppressive maintenance regimen or HT period. Univariate analysis indicates that the only induction agent increasing the risk of lymphoma was ATG (Table 3); risk was not increased by either OKT3 or IL-2R blockers. In a retrospective review of $154 \mathrm{HT}$ patients, Swinnen et $\mathrm{al}^{4}$ observed the opposite trend: the incidence of PTLD was 9-fold higher among OKT3- treated patients than among others (most of whom had received ATG induction therapy). The difference between our results and those of Swinnen and colleagues is likely attributable to the use of different OKT3 doses: their OKT3 induction regimen was $5 \mathrm{mg} /$ day for 14 days; the mean duration of OKT3 induction in the present study was 7.8 days (SD 3.9 days) and, in general, Spanish centers reduced the dose and/or duration of OKT3 induction during the 1990s. ${ }^{10,11}$ A sharp post-1990 fall in the incidence of lymphoma among OKT3-induced patients in the CTS database has similarly been attributed to probable reductions in dose and treatment duration. ${ }^{9}$

Although the utility of induction therapy after HT is still a matter of debate, ${ }^{12-15}$ it is used in many centers because it allows delayed institution of immunosuppression with calcineurin inhibitors, and therefore spares renal function. According to the latest ISHLT report, ${ }^{8}$ around $50 \%$ of patients now receive induction therapy, mainly with IL-2R blocking antibodies and polyclonal ATG; the use of OKT3 has been decreasing. Induction therapy, if it is a risk factor, is in principle an avoidable one. Induction agents did not emerge as a risk factor in one study of a subset of ISHLT cases evaluated by O'Neill and colleagues. ${ }^{16}$ The proportion of our patients given some kind of induction therapy, 61\%, is about 1.3 times the ISHLT average for the period 1996 to $2004,{ }^{8}$ and about 1.5 times the proportion in O'Neill et al's study ${ }^{16}(37 \%)$, but does not appear to have led to a greater incidence of lymphomas. It is necessary, however, to distinguish among different induction agents: in the present study, induction with IL-2R blockers did not increase the risk of PTLD, and we know of no reports of them doing so.

Since the early 1990s, anti-viral agents have been administered in many centers to transplant patients prophylactically during the first 3 months to reduce the risk of herpesvirus disease, especially cytomegalovirus (CMV) disease. For CMV, prophylaxis appears to be successful. ${ }^{17}$ Also, prophylaxis with ganciclovir has been reported to reduce the risk of PTLD in kidney transplant patients. ${ }^{18}$ In this study we found that prophylaxis with ganciclovir or acyclovir significantly reduced the risk of post-HT lymphoma. More specifically, multivariate analyses showed that, in the absence of anti-viral prophylaxis, induction with ATG and induction with OKT3 both increased the incidence of lymphomas, whereas risk was not increased by any induction treatment if anti-viral prophylaxis was employed (either acyclovir in the first 3 months after HT, or ganciclovir in the first month). How acyclovir and ganciclovir achieve this reduction in risk is unknown; the investigators in the kidney transplant study suggested that, because both acyclovir and ganciclovir act only during the lytic stage of infection, their efficacy for anti-viral prophylaxis must be due to action during the initial infection with EBV or during re-activation of a latent infection. ${ }^{18}$ To our knowledge, the present study is the first to assess the influence of antiviral prophylaxis on whether induction therapy increases the risk of post-HT lymphoma.

The most obvious limitation of this study is the paucity of data on EBV serology in the SPHTTR (these data are available for only $27 \%$ of patients), which prevented investigation of whether the efficacy of anti-viral prophylaxis in reducing the risk of PTLD depends on EBV status. 
In conclusion, among Spanish HT patients, induction therapy other than with IL-2R antagonists has in general significantly increased the risk of lymphomas, except when acyclovir or ganciclovir were administered prophylactically after HT. If induction with OKT3 or antithymocyte globulin is utilized, anti-viral prophylaxis should also be employed to reduce the risk of post-HT lymphoma.

The authors are grateful to the researchers and staff at all the Spanish heart transplant centers that contributed data to this study. We also thank Zulaika Grille for coordination of the SPHTT Registry, Soly Santiago for statistical analyses and preparing tables and figures, and Francisco Arnal for advice on pathologic studies. Finally, we thank Ian-Charles Coleman for helpful criticism and for the English version of this report.

\section{REFERENCES}

1. Newell KA, Alonso EM, Whitington PF, et al. Posttransplant lymphoproliferative disease in pediatric liver transplantation. Interplay between primary Epstein-Barr virus infection and immunosuppression. Transplantation 1996;62:370-5.

2. Thorley-Lawson D, Gross A. Persistence of the Epstein-Barr virus and the origins of associated lymphomas. N Engl J Med 2004;350: 1328-37.

3. Opelz G, Schwarz V, Henderson R, Kneifel G, Ruhenstroth A. Non-Hodgkin's lymphoma after kidney or heart transplantation: frequency of occurrence during the first posttransplant year. Transplant Int 1994;7(suppl 1):S353- 6.

4. Swinnen L, Costanzo-Nordin M, Fisher S, et al. Increased incidence of lymphoproliferative disorder after inmunosuppression with the monoclonal antibody OKT3 in cardiac transplant recipients. N Engl J Med 1990;323:1723-8.

5. Ratkovec R, O’Connell J, Bristow M, et al. Post-transplant lymphoproliferative disease in cardiac transplant patients receiving OKT3 therapy. Clin Transplant 1992;6:260-4.

6. Gao SZ, Chaparro SV, Perlroth M, et al. Post-transplantation lymphoproliferative disease in heart and heart-lung transplant recipients: 30-year experience at Stanford University. J Heart Lung Transplant 2003;22:505-14.

7. Peraira JR, Segovia J, Fuertes B, et al. Current induction immunosuppression and post- heart transplant lymphoproliferative disorders. Transplant Proc 2003;35:2009-10.

8. Taylor D, Edwards L, Boucek M, et al. Registry of the International Society for Heart and Lung Transplantation: twenty-third official adult heart transplantation report-2006. J Heart Lung Transplant 2006;25:869-79.

9. Opelz G, Dohler B. Lymphomas after solid organ transplantation: a collaborative transplant study report. Am J Transplant 2003;4: 222-30.

10. Rodriguez J, Crespo-Leiro M, Paniagua M, et al. Induction of immunosuppression with OKT3 following heart transplantation: kidney function as a criterion for control of protocol duration. Transplant Proc 1999;31:2517-8.

11. Alonso-Pulpón L, Serrano-Fiz S, Rubio J, et al. Efficacy of low-dose OKT3 as cytolytic induction therapy in heart transplantation. J Heart Lung Transplant 1995;14:136-42.

12. Uber PA, Mehra MR. Induction therapy in heart transplantation: is there a role? J Heart Lung Transplant 2007;26:205-9.

13. Higgins R, Kirklin J, Brown R, et al. To induce or not to induce: do patients at greatest risk for fatal rejection benefit from cytolytic induction therapy? J Heart Lung Transplant 2005;24: 392-400.

14. Hauptman PJ, Mehra MR. It is time to stop ignoring malignancy in heart transplantation: a call to arms? J Heart Lung Transplant 2005;24:1111-3.

15. Radovancevic B, Konuralp C, Vrtovec B, et al. Factors predicting 10-year survival after heart transplantation. J Heart Lung Transplant 2005;24:156-9.

16. O’Neill JO, Edwards LB, Taylor DO. Mycophenolate mofetil and risk of developing malignancy after orthotopic heart transplantation: analysis of the transplant registry of the International Society for Heart and Lung Transplantation. J Heart Lung Transplant 2006;25:1186-91.

17. Hodson EM, Jones CA, Webster AC, et al. Antiviral medications to prevent cytomegalovirus disease and early death in recipients of solid-organ transplants: a systematic review of randomised controlled trials. Lancet 2005;365:2105-15.

18. Funch DP, Walker AM, Schneider G, Ziyadeh NJ, Pescovitz MD. Ganciclovir and acyclovir reduce the risk of post-transplant lymphoproliferative disorder in renal transplant recipients. Am J Transplant 2005;5:2894-900. 


\section{APPENDIX}

The following investigators also participated in the Spanish Post-Heart Transplant Tumour Registry: Maria J. Paniagua and Jose A Rodriguez (Complejo Hospitalario Universitario Juan Canalejo, La Coruña); Javier Segovia and Manuel Gomez- Bueno (Clinica Puerta de Hierro, Madrid); Carmen Segura Saint-Gerons (Hospital Universitario Reina Sofia, Córdoba); Luis Martinez-Doltz (Hospital Universitario La Fe, Valencia); Miguel Angel Gómez-Sanchez and Pilar Escribano (Hospital Universitario 12 de Octubre, Madrid); Juan Fernández-Yáñez (Hospital General Universitario Gregorio Marañón, Madrid); Josep Roca (Hospital Universitario de Bellvitche, Barcelona); Matias Ubilla (Clínica Universitaria de Navarra, Pamplona); Maria Martin-Fernández and Jose L. Rodriguez-Lambert (Hospital Universitario Central de Asturias, Oviedo); Félix Perez- Villa (Hospital Clinic i Provincial, Barcelona); Iris P. Garrido (Hospital Universitario Virgen de la Arrixaca, Murcia); Maria Luisa Sanz-Julve (Hospital Universitario Miguel Server, Zaragoza); Javier LópezDiaz (Hospital Clinico Universitario, Valladolid); Vicens Brossa and Sonia Mirabet (Hospital de la Santa Creu i San Pau, Barcelona); and Francisco Gonzalez-Vichez (Hospital Universitario Marqués de Valdecilla, Santander). 